\title{
Self-complementary adeno-associated virus 5-mediated gene transduction of a novel CD40L mutant confers direct antitumor effects in lung carcinoma
}

\author{
WEI XU, YUANYUAN XU, YUNYAN WEI, YAOXI TAN, HONGYE ZHAO, WEIHONG ZHAO and JIANQING WU \\ Department of Geriatrics, The First Affiliated Hospital of Nanjing Medical University, Nanjing, Jiangsu 210029, P.R. China
}

Received February 7, 2014; Accepted September 24, 2014

DOI: $10.3892 / \mathrm{mmr} .2014 .2765$

\begin{abstract}
CD40 ligand (CD40L) gene therapy offers a potentially useful option for lung cancer due to its multiple antitumor activities. However, membrane-bound CD40L may be proteolytically cleaved to form soluble CD40L (sCD40L), which results in adverse effects. In a previous study by our group, it was demonstrated that recombinant self-complementary adeno-associated virus 5 (scAAV5) efficiently delivered genes to lung cancer cells. In the present study, an scAAV5 expressing a non-cleavable human CD40L mutant (scAAV5-CD40L-M) was generated and its direct antitumor effects in lung cancer were evaluated. Transduction with scAAV5-CD40L-M resulted in effective expression of CD40L on the cell surface with low levels of cleaved sCD40L, which significantly reduced the percentage of viable cells and promoted caspase-3-dependent apoptosis of CD40-positive lung carcinoma A549 cells, compared with scAAV5-CD40L transduction $(\mathrm{P}<0.05)$. Furthermore, treatment with scAAV5-CD40L-M exerted a significant antitumor effect against CD40-positive A549 xenografts by inducing apoptosis $(\mathrm{P}<0.05)$ with few side effects. Gene therapy using an scAAV5 vector expressing non-cleavable human CD40L mutant may therefore have direct antitumor effects against CD40-positive lung cancers. These tumoricidal effects of scAAV5-CD40L-M treatment make it a promising therapeutic technique for the treatment of lung cancer.
\end{abstract}

\section{Introduction}

Lung cancer is a leading cause of global cancer-associated mortality (1). Non-small cell lung cancer (NSCLC) accounts

Correspondence to: Professor Jianqing Wu, Department of Geriatrics, The First Affiliated Hospital of Nanjing Medical University, 300 Guangzhou Road, Nanjing, Jiangsu 210029, P.R. China

E-mail: jwuny@njmu.edu.cn

Key words: self-complementary adeno-associated virus 5, CD40L mutant, gene therapy, lung cancer for $\sim 80 \%$ of all types of lung cancer. Although recent advances have been made in the development of diagnosis and treatment strategies, the prognosis of patients with NSCLC remains poor (2).

Immunogene therapy using immunostimulatory molecules to enhance anti-tumor immunity offers a promising therapeutic option for lung cancer (3). The CD40 ligand (CD40L), a type II membrane-bound protein belonging to the tumor necrosis factor (TNF) superfamily, was used in cancer gene therapy due to its ability to trigger Th1-type immune responses that drive effector cells, including cytotoxic T lymphocytes, natural killer cells and M1 macrophages, which normalize the tumor microenvironment and directly suppress the growth of certain CD40-positive tumors (4). Novel therapies based on CD40L, including infusion of recombinant CD40L protein, anti-CD40 antibodies and CD40L gene therapy, potentially provide numerous anti-cancer approaches in one therapy and offer an attractive option for clinical trials (5-7).

Several studies indicated that the precise form of the CD40 stimulus, inducing a membrane-bound and soluble version of CD40L (sCD40L), influenced the therapeutic responses in carcinoma cells. sCD40L is shed from membrane-bound forms via matrix metalloproteinase (MMP) cleavage and provides local cytokine-like amplification of CD40 signaling (8). A study indicated that sCD40L induced pro-survival and pro-apoptotic signals in CD40-positive solid carcinoma cells (9). In clinical trials, elevated serum levels of sCD40L were found in autoimmune diseases, metabolic and cardiovascular diseases and the cytokine-like effects of SCD40L may contribute to the pathogenesis of these disorders by acting on CD40(+) bystander cells $(10,11)$. Membrane-bound CD40L, however, through sustained activation of TNF receptor-associated factor 3 (TRAF3)-dependent c-Jun N-terminal kinase signals and suppression of TRAF6-dependent survival signals, was able to directly induce cell death whilst creating few side effects (12). Therefore, as a potential anti-cancer therapeutic, membrane-bound CD40L presents a more attractive option than its soluble form.

To circumvent the possible stimulating proliferative activity and adverse inflammatory effects caused by sCD40L, a membrane-stable mutant form of human CD40L (CD40L-M) was designed and engineered. As a means of delivering 
CD40L-M for clinical application, the transduction efficiency of various serotypes of adeno-associated virus (AAV) vectors in an NSCLC cell line were examined. The efficient recombinant self-complementary AAV5 (scAAV5) was subsequently selected as a therapeutic vehicle (11). An scAAV5 encoding human CD40L mutant (AAV5-CD40L-M), which results in consistent expression of ligand at the cell membrane, was generated. The direct anti-tumor effects of cleavage-resistant CD40L delivered by scAAV5 were examined in vitro and in vivo.

\section{Materials and methods}

Cell culture. The human NSCLC cell lines A549 and H1650 and the human embryonic kidney cell line HEK293 (293T) were obtained from the Cell Resource Center (Shanghai, China). The cells were maintained in RPMI-1640 medium (Gibco, Carlsbad, CA, USA) supplemented with $10 \%$ fetal bovine serum (Gibco-BRL, Grand Island, NY, USA).

ConstructionofscAAV5vectors.Plasmids pdsAAV-CB-CD40L and pdsAAV-CB-CD40L-M, which are self-complementary double-sequence AAV plasmids containing human wild-type CD40L cDNA and CD40L-M cDNA (containing six substitutions: Gln114 to Pro114, Lys115 to Arg115, Asp117 to Glu117, Gln118 to Glu118, Asn119 to Asp119 and Pro120 to Ser120), were constructed by Invitrogen Life Technologies (Carlsbad, CA, USA) $(13,14)$. AAV vectors were generated as described by the Penn Vector Core (http://www.med.upenn. edu/gtp/vector_core/production.shtml). Briefly, a plasmid encoding the transgene of interest, expressed from a cytomegalovirus enhancer/ $\beta$-actin (CB) promoter, flanked by AAV2 inverted terminal repeats, was packaged by triple transfection of HEK293 cells with plasmids encoding the AAV2 rep gene and the AAV5 cap gene and an adenovirus helper plasmid (Fig. 1A). Vectors were purified by $\mathrm{CsCl}$ ultracentrifugation and titers were determined by slot blot analysis, as previouslt described (15).

Quantitative polymerase chain reaction ( $q P C R)$. PCR was performed using the SYBR Green RT-PCR kit (Toyobo Co., Ltd., Osaka, Japan) according to the manufacturer's instructions. The PCR primer pairs used were as follows: CD40L (NM-000074, position 488-620, 132 bp fragment) forward, 5'-TGA GCA ACA ACT TGG TAA CCC TGG-3' and reverse, 5'-CTG GCT ATA AAT GGA GCT TGA CTC G-3'; $\beta$-actin (NM-001101, position 939-1124, 205 bp fragment) forward, 5'-TGA CGT GGA CAT CCG CAA AG-3' and reverse, 5'-CTG GAA GGT GGA CAG CGA GG-3' (Invitrogen Life Technologies).

Flow cytometric analysis. CD40L and CD40 expression was determined by flow cytometry (FACSCalibur; BD Biosciences, Franklin Lakes, NJ, USA) using an allophycocyanin (APC)-conjugated CD154 antibody (Ab) diluted at 1:25 or phycoerythrin (PE)-conjugated CD40 Ab diluted at 1:50. Appropriate APC- or PE-conjugated isotype control was used to measure background staining. Annexin V-fluorescein isothiocyanate (FITC) and/or propidium iodide (PI) staining (TACS Annexin V-FITC kit; Trevigen, Gaithersburg, MD,
USA) was used to detect apoptotic cells. Caspase-3 inhibitor (Ac-DEVD-CHO; Santa Cruz Biotechnology, Inc., Dallas, TX, USA) was employed in a competition experiment.

Cell viability assay. Cell viability was determined by MTT assay using a Cell Proliferation Kit I (Roche Diagnostics $\mathrm{GmbH}$, Mannheim, Germany). Cancer cells were seeded into 96-well plates. After incubation, the medium was replaced with $50 \mu \mathrm{l}$ MTT reagent $(2 \mathrm{mg} / \mathrm{ml})$ followed by further incubation in an incubator for $2 \mathrm{~h}$. Next, the medium was removed and dimethylsulfoxide $(150 \mu \mathrm{l})$ was added to each well. The absorbance at $560 \mathrm{~nm}$ was measured with a spectrophotometer (PerkinElmer, Waltham, MA, USA). A competition experiment with anti-human CD40L monoclonal antibody (100 ng/l; BioLegend, Inc., San Diego, CA, USA) was performed.

Western blot analysis. Briefly, proteins were separated by $6-15 \%$ SDS-PAGE and transferred to polyvinylidene difluoride membranes (Hybond-P; GE Healthcare, Little Chalfont, UK). The primary antibodies used were: Rabbit anti-caspase-3 polyclonal antibody (pAb; 1:100; sc-7148) and rabbit anti- $\beta$-actin pAb (1:1,000; sc-130656; Santa Cruz Biotechnology, Inc.). The secondary antibodies were horseradish peroxidase-conjugated antibodies against rabbit immunoglobulin G (GE Healthcare, Waukesha, WI, USA). Immunoreactive bands in the western blots were visualized using enhanced chemiluminescence substrates (ECL Plus; GE Healthcare).

Caspase-3 activity assay. The activity of caspase-3 was evaluated using the Caspase-3 Activity Assay kit (C115; Beyotime Institute of Biotechnology, Haimen, China) according to the manufacturer's instructions. The caspase-3 activity assay is based on spectrophotometric detection of the chromophore $p$-nitronanilide (pNA), following its cleavage from the labeled substrate. The release of pNA was quantified by determining the absorbance using a Sunrise $^{\mathrm{TM}}$ microplate reader (Tecan Group, Ltd., Männedorf, Switzerland) at $405 \mathrm{~nm}$.

ELISA. The concentration of $\mathrm{sCD} 40 \mathrm{~L}$ in supernatants and serum following treatment were measured using a human sCD40L ELISA kit (ELH-CD40L-001; RayBiotech, Inc., Norcross, GA, USA) according to the manufacturer's instructions.

Mouse model studies. Xenografts were prepared by implanting tumor fragments derived from A549 cells $\left[5 \times 10^{6}\right.$ cells suspended in $100 \mu 1$ phosphate-buffered saline (PBS)] subcutaneously into the back of six-week-old male BALB/c nude mice. The mice were obtained from the animal center at Nanjing Medical University (Nanjing, China). All mice were maintained on a diet of standard rodent chow and water supplied ad libitum, with a regular light-dark cycle and at $23^{\circ} \mathrm{C}$. When the tumor volume reached $\sim 100 \mathrm{~mm}^{3}$, the mice were randomly divided into four groups (six mice/group). Intratumoral injection with $0.5 \mathrm{ml}$ scAAV5-GFP, scAAV5-CD40L and scAAV5-CD40L-M vectors, respectively, [at 1x1,011 viral genomes (vgs)/mouse] or $0.5 \mathrm{ml}$ PBS was performed.. Tumor 
growth was subsequently monitored every three days for 30 days by measuring tumor size with a caliper. The tumor volume was calculated using the following formula: Tumor volume $=$ length $\mathrm{x}$ width ${ }^{2} \times 0.5$. All animal experiments were performed in accordance with the Guide for the Care and Use of Laboratory Animals of the National Institutes of Health. The protocol was approved by the Committee on the Ethics of Animal Experiments of Nanjing Medical University (permit number, 307005).

Immunohistochemistry. Immunohistochemistry using the rabbit anti-CD40L antibody (ab65854; Abcam, Cambridge, UK) was employed to investigate paraffin wax-embedded tissue sections using a routine avidin-biotin-immunoperoxidase technique (Histostain-Plus kit, Zymed, San Franzisco, USA). For quantitative analysis of apoptosis, sections were assayed by the terminal deoxynucleotidyl transferase-mediated dUTP-FITC nick end-labeling (TUNEL) method using a One Step TUNEL Apoptosis Assay kit (C1086; Beyotime). The number of positive cells was counted in ten random fields using microscopy (magnification, x100; LSM 700; Carl Zeiss, Oberkochen, Germany).

Statistical analysis. The two-tailed Student's t-test application of Microsoft Excel 2013 (Microsoft Corporation, Redmond, WA, USA) was used for all continuous outcome variables, including tumor size and weight. A repeated Student's t-test was used to compare the treated groups with the control group. Values are presented as the mean \pm standard deviation and $\mathrm{P}<0.05$ was considered to indicate a statistically significant difference between values.

\section{Results}

Expression of scAAV5-CD4OL-M. The expression cassette containing the CD40L or CD40L-M cDNA was inserted into the cloning site of pdsAAV-CB-GFP to create the expression plasmid pdsAAV-CB-CD40L or pdsAAV-CB-CD40L-M, respectively. The correct plasmids were identified by sequencing. Recombinant scAAV5-CD40L and scAAV5-CD40L-M vectors (Fig. 1A) were generated as aforementioned. These viruses were used to infect the A549, H1650 and 293T cell lines. Expression of CD40 was detected in the A549 cell line $(65.63 \pm 6.02 \%)$, but not in the H1650 or $293 \mathrm{~T}$ cell lines. Infection with the equivalent multiplicity of infection (MOI, $10^{4} \mathrm{vgs} / \mathrm{cell}$ ) of CD40L-M resulted in membranous ligand expression determined by flow cytometry (Fig. 1B). Expression of CD40L and CD40L-M was confirmed by qPCR analysis (Fig. 1C).

SCAAV5-CD4OL-M inhibits the proliferation of CD40-positive cell lines. The direct growth-inhibitory activities of scAAV5-CD40L and scAAV5-CD40L-M were assessed by MTT assay using CD40-positive A549 and CD40-negative H1650 lung cancer cell lines. Significantly decreased A549 viability was observed (Fig. 2A) following scAAV5-CD40L or scAAV5-CD40L-M transduction at $96 \mathrm{~h}$ (MOI of $10^{4} \mathrm{vgs} / \mathrm{cell}$ : scAAV5-GFP, $85.02 \pm 7.34 \%$; scAAV5-CD 40L, 49.53 $\pm 6.05 \%$; scAAV5-CD 40L-M, $23.56 \pm 3.67 \%$; MOI of $10^{5} \mathrm{vgs} / \mathrm{cell}$ : scAAV5-GFP,
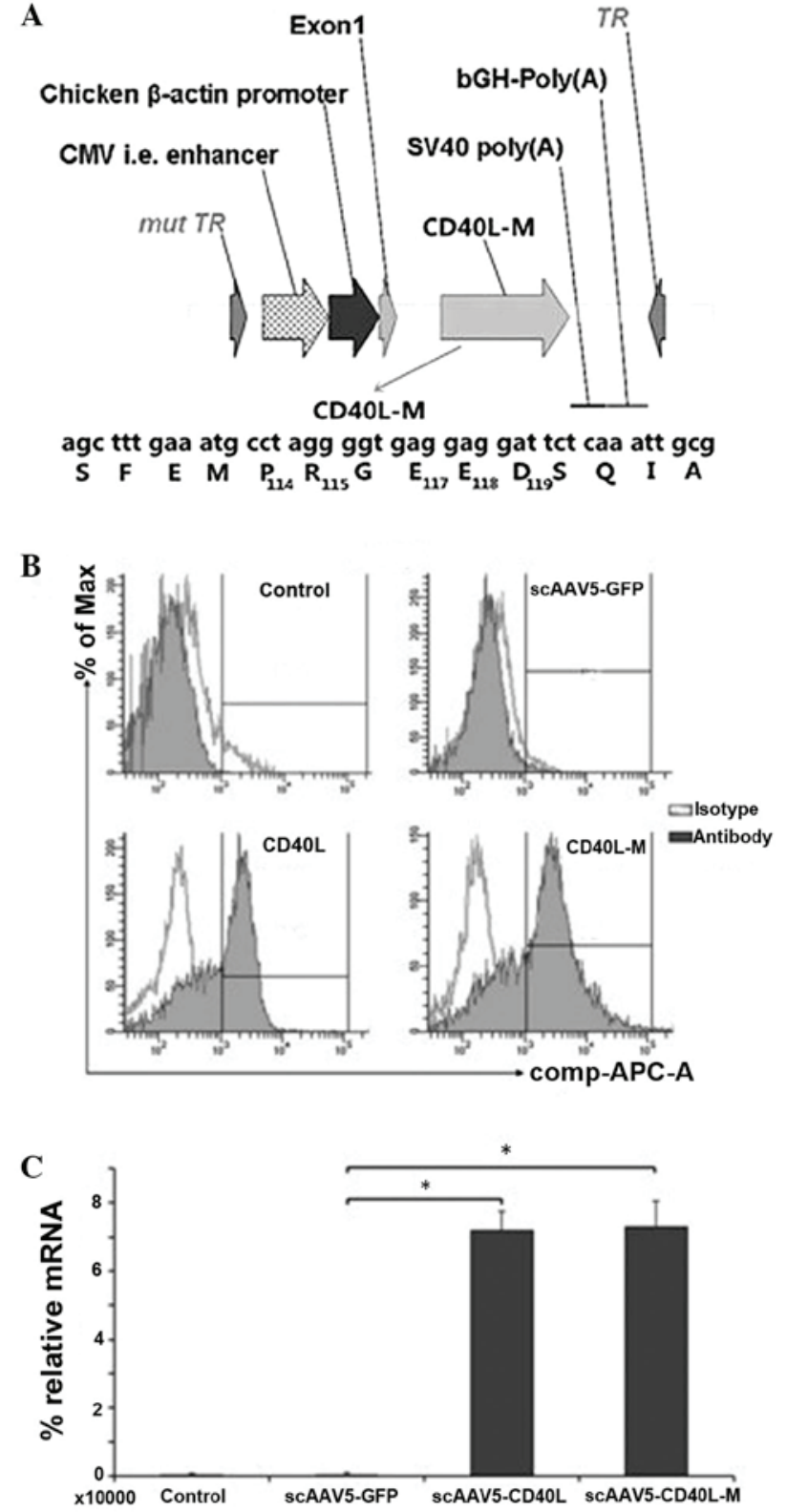

Figure 1. Maps of scAAV-CD40L-M and CD40L expression following transduction. (A) Schematic representation of the scAAV genome containing a mutated 5 inverted terminal repeat and an actin intron from which 722 nucleotides were deleted. This construct contained the cytomegalovirus enhancer/ $\beta$-actin promoter driving expression of CD40L-M, including six mutation sites. (B) scAAV5-delivered CD40L was expressed on the cell membrane of A549 cells. (C) Relative CD40L gene transcripts evaluated by quantitative polymerase chain reaction in the A549 cell line. Values are expressed as the mean \pm standard deviation of triplicate samples, ${ }^{*} \mathrm{P}<0.05$. scAAV, self-complementary adeno-associated virus; CD40L-M, CD40 ligand mutant; GFP, green fluorescent protein.

$83.76 \pm 8.54 \% ; \quad$ scA AV $5-\mathrm{CD} 40 \mathrm{~L}, \quad 35.37 \pm 4.78 \%$; scAAV5-CD40L-M, 18.92 22.03\%; versus scAAV5-green fluorescent protein (GFP); $\mathrm{P}<0.01)$. A significant difference between the viability of scAAV5-CD40L/A549 cells and that of scAAV5-CD40L-M/A549 cells was also distinguishable $(\mathrm{P}<0.05)$. This inhibitory effect was attenuated when cells were pre-incubated with an anti-CD40L monoclonal antibody. By contrast, no difference was observed in the CD40-negative H1650 cells following transduction (Fig. 2B). 

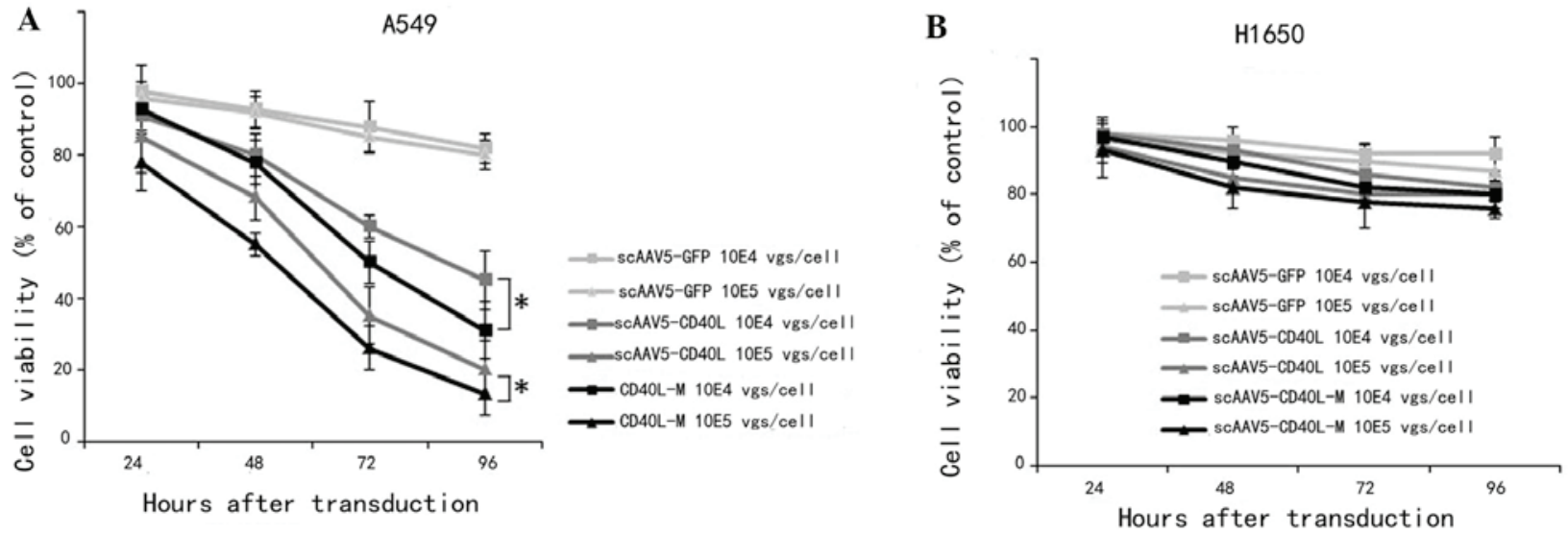

Figure 2. Cell viability following transduction. Cell viability was evaluated by MTT assay following transduction with recombinant scAAV5-CD40L-M vectors at a multiplicity of infection of $10^{4} \mathrm{vgs} / \mathrm{cell}$ and $10^{5} \mathrm{vgs} / \mathrm{cell}$, in comparison to that of scAAV5-CD40L-treated cells. (A) CD40-positive lung carcinoma A549 cells. (B) CD40L-negative H1650 cells. Values are expressed as the mean \pm standard deviation of triplicate samples, "P $<0.05$. scAAV5, self-complementary adeno-associated virus 5; CD40L-M, CD40 ligand mutant.
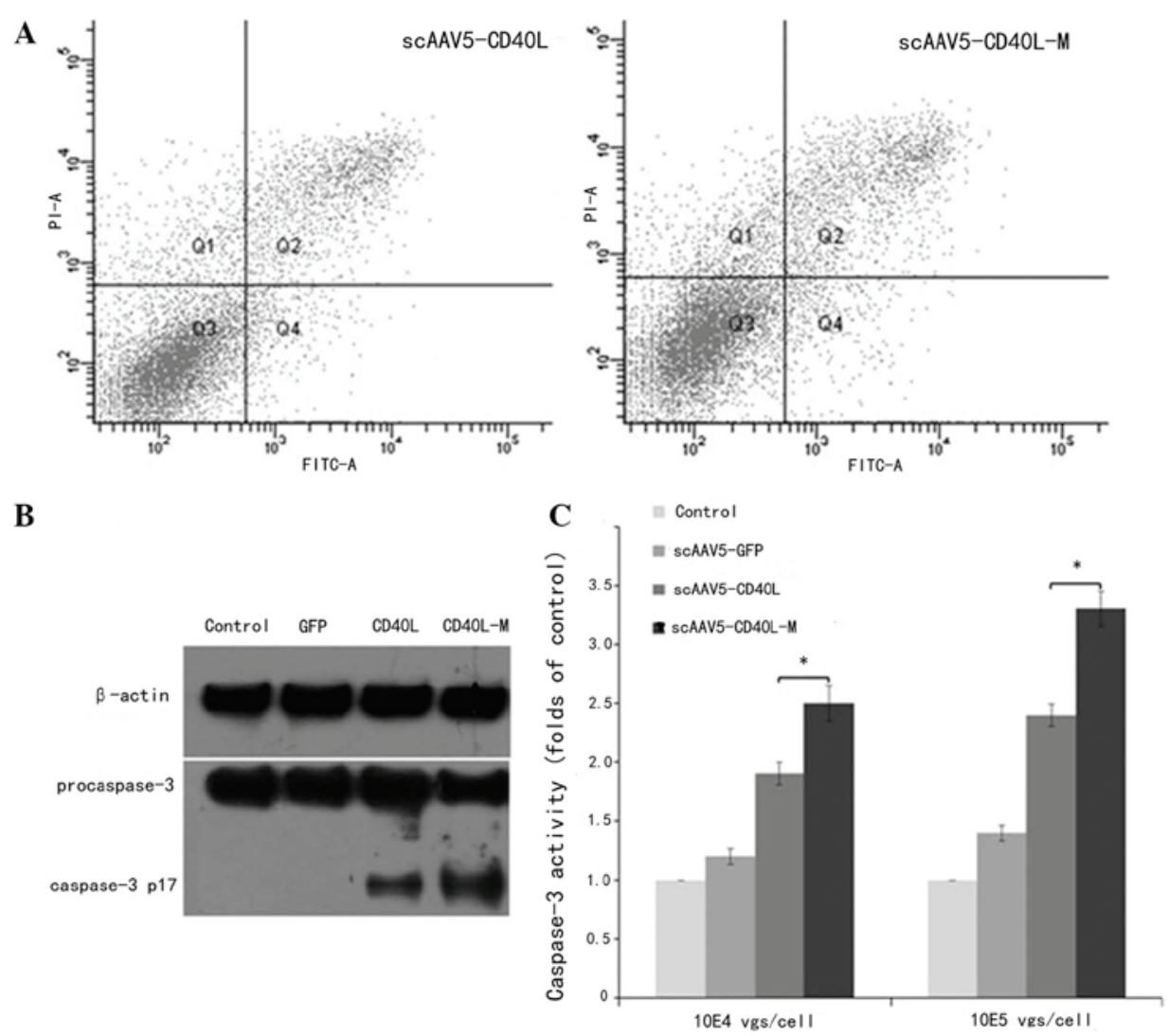

Figure 3. scAAV5-CD40L-M induces apoptosis in CD40-positive lung carcinoma A549 cells. (A) Flow cytometric analysis of Annexin V-FITC/PI staining in A549 cells $48 \mathrm{~h}$ following transduction with viral vectors at a multiplicity of infection of $10^{4} \mathrm{vgs} / \mathrm{cell}$. scAAV5-CD40L and scAAV5-CD40L-M induced apoptosis of CD40-positive A549 cells with apoptotic rates of 17.83 \pm 1.32 and $12.56 \pm 1.05 \%$, respectively. (B) Cell lysates from vehicle-treated cells were evaluated by western blot analysis for the processing of caspase- 3 and (C) by enzymatic assay for caspase- 3 activity. Values are expressed as the mean \pm standard deviation of triplicate samples, "P<0.05. scAAV5, self-complementary adeno-associated virus; FITC, fluorescein isothiocyanate; PI, propidium iodide; GFP, green fluorescent protein; CD40L-M, CD40 ligand mutant; vgs, viral genomes.

scAAV5-CD40L-M induces CD40-positive A549 cell apoptosis. Relative to CD40-negative H1650 cells, scAAV5-CD40L and scAAV5-CD40L-M $\left(10^{4} \mathrm{vgs} / \mathrm{cell}\right)$ were found to induce CD40-positive A549 cell apoptosis as indicated by Annexin V-FITC/PI double staining with apoptotic rates of
$17.83 \pm 1.32$ and $12.56 \pm 1.05 \%$, indicating a significant difference between the two groups $(\mathrm{P}<0.05 ;$ Fig. $3 \mathrm{~A})$.

To investigate whether the apoptotic effect observed was associated with caspase- 3 activation, western blot analysis was used to examine the expression levels of cleaved caspase- 3 in 
A

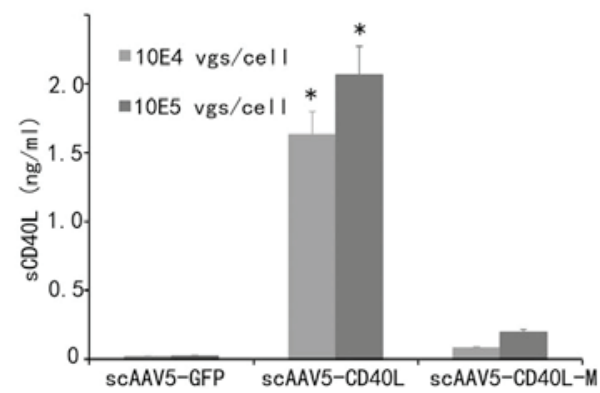

B

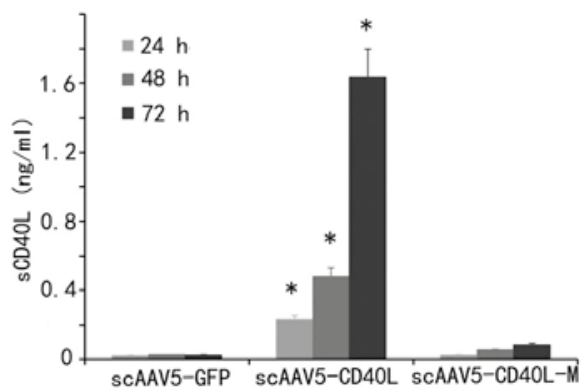

Figure 4. sCD40L expression was evaluated in cell-free supernatants of A549 cells post-infection by ELISA. (A) Samples were collected from the culture media $48 \mathrm{~h}$ post-infection at MOI $10^{4} \mathrm{vgs} / \mathrm{cell}$ and $10^{5} \mathrm{vgs} / \mathrm{cell}$. (B) Samples were tested at 24, 48 and $72 \mathrm{~h}$ following transduction at MOI of $10^{4} \mathrm{vgs} / \mathrm{cell}$. Values are expressed as the mean \pm standard deviation of triplicate samples. ${ }^{*} \mathrm{P}<0.01$ vs. corresponding scAAV5-CD40L-M transductants. scAAV5, self-complementary adeno-associated virus 5; MOI, multiplicity of infection; GFP, green fluorescent protein; sCD40L, soluble CD40 ligand; M, mutant; vgs, viral genomes.

A549 cells. The expression levels of cleaved caspase-3 protein in scAAV5-CD40L-M-infected cells were higher than those in scAAV5-CD40L-infected cells (10 vgs/cell; Fig. 3B). Caspase-3 activity was further analyzed by enzymatic assay. The level of caspase- 3 activation was markedly higher in cells transduced with scAAV5-CD40L-M for $48 \mathrm{~h}$ than that of cells exposed to scAAV5-CD40L or AAV5-GFP (Fig. 3C). Apoptosis was not induced when the cells were pre-incubated with caspase-3 inhibitor (data not shown). These results indicated that CD40L-associated cell death may be mediated by a caspase-dependent signaling pathway.

sCD40L secretion is significantly decreased in scAAV5-CD40L-M-transduced cells. Secretion of sCD40L following transduction was detected in supernatants to examine whether CD40L-M was resistant to cleavage on the cell surface. As indicated in Fig. 4A and B, the amount of sCD40L from the culture supernatants of scAAV5-CD40L-transduced cells was significantly increased following transduction compared with that of the corresponding mock and CD40L-M groups $(\mathrm{P}<0.01)$.

Antitumor activity of ScAAV5-CD4OL-M against lung cancer xenografts. To focus on the direct tumor growth-inhibitory features of AAV5-CD40L-M, in vivo analyses were performed via lung cancer heterotransplants in athymic nude mice. scAAV5-CD40L and scAAV5-CD40L-M each effectively decreased the tumor growth rate (Fig. 5A). The mean tumor weights were $0.43 \pm 0.03,0.31 \pm 0.02$ and $0.29 \pm 0.03 \mathrm{~g}$ in the scAAV5-GFP, scAAV5-CD40L and scAAV5-CD40L-M groups, respectively (Fig. 5B).

CD40L expression was detected in the scAAV5-CD40L- and scAAV5-CD40L-M-injected tumors but not in the scAAV5-GFP-injected tumors (Fig. 5C). The effect of scAAV5-CD40L-M treatment on apoptosis was assessed using an in situ TUNEL assay. The most significant level of apoptosis was observed in the tumors of mice receiving scAAV5-CD40L- or scAAV5-CD40L-M-treatment, compared with that of scAAV5-GFP mice (Fig. 5D; $\mathrm{P}<0.05$, respectively).

The systemic toxicity of scAAV5-CD40L-M treatment was examined. Histopathological studies indicated no apparent lesions in the lung, liver, spleen or kidney. Levels of SCD40L in serial serum samples were $\sim 0$. Serum alanine transaminase, aspartate aminotransferase, serum urea nitrogen and creatinine were determined. No significant difference was detected between groups (Data not shown).

\section{Discussion}

CD40L transgene expression in human CD40-positive solid carcinoma cells was found to produce a direct growth-inhibitory effect through apoptotic induction and/or cell cycle blockage (16-22). However, sCD40L cleaved from membrane-bound CD40L may regulate CD40L activity by downregulating surface CD40L and quenching CD40L with an sCD40L 'decoy' (23), which is unable to induce survival signals in cancer cells (24) or enter into the systemic circulatory system and cause inflammatory diseases $(10,11)$. Therefore, in the present study, to reduce the adverse effects caused by SCD40L, a human CD40L mutant that was resistant to proteolytic cleavage was generated as a candidate gene for immunogenetic therapy of lung cancer.

Successful gene therapy is dependent upon the efficiency and availability of gene delivery systems. Recombinant AAVs have been widely used for gene delivery in animal models and their use in human gene therapy is currently being evaluated in clinical trials (http://www.abedia.com/wiley/). However, limitations in vector tropism, including limited tissue specificity and insufficient transduction efficiencies, preclude therapeutic applications in certain tissues (25). To achieve greater efficacy of AAV-gene therapy for lung carcinoma, the transduction efficiency of various AAV vector serotypes in NSCLC cell lines were examined. The results demonstrated that scAAV5 vectors, which bypass the requirement of conventional viral second-strand DNA synthesis, are the most robust to deliver the gene in A549 cells (13). Recently, a study verified that $\mathrm{N}$-linked sialic acid on the surface of lung cancer cells mediates tropism of vectors based on AAV5 (26). Therefore, based on previous studies, a recombinant scAAV5 expressing mutant CD40L was generated in the present study to further explore the direct antitumor effects of wild-type and cleavage-resistant CD40L in vitro and in vivo.

The results of the present study indicated that scAAV5-CD40L-M produced a more profound growth-inhibitory effect in CD40-positive cells compared with that in wild-type scAAV5-CD40L. Caspase-3 activation assay and Annexin $\mathrm{V}$ staining confirmed that CD40L-M was a more 
A

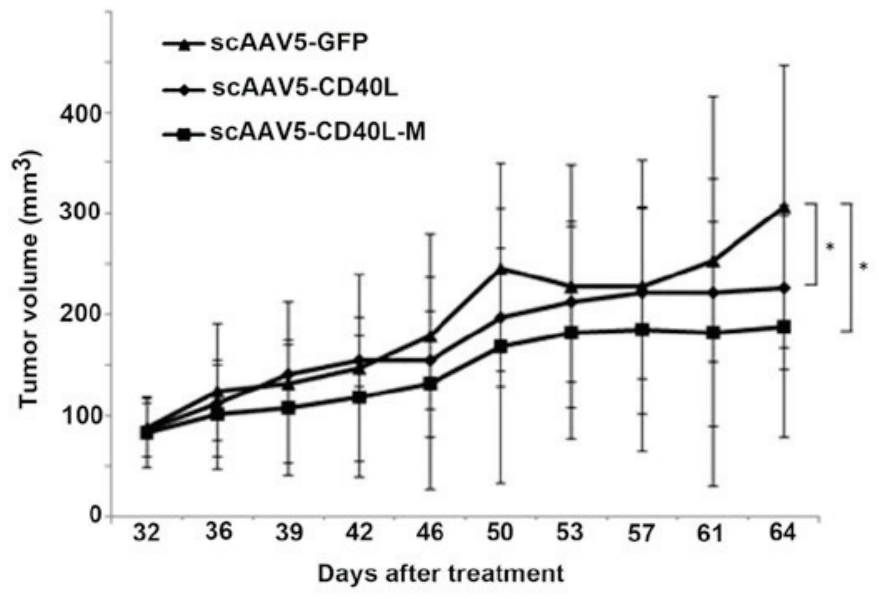

B

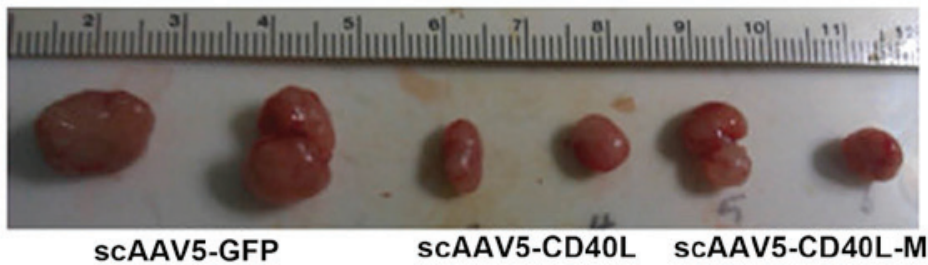

C

ScAAV5-GFP

ScAAV5-CD40L

ScAAV5-CD40L-M
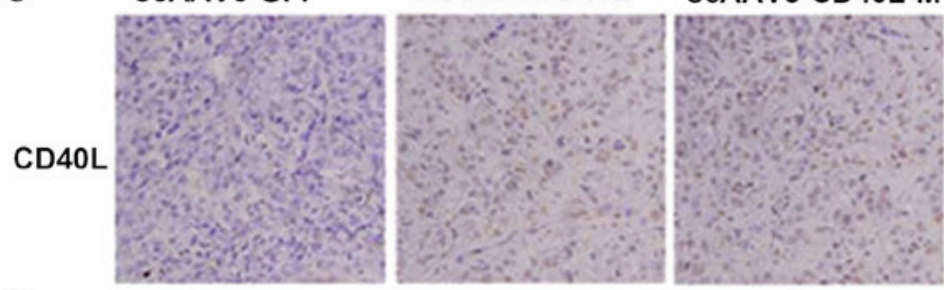

D
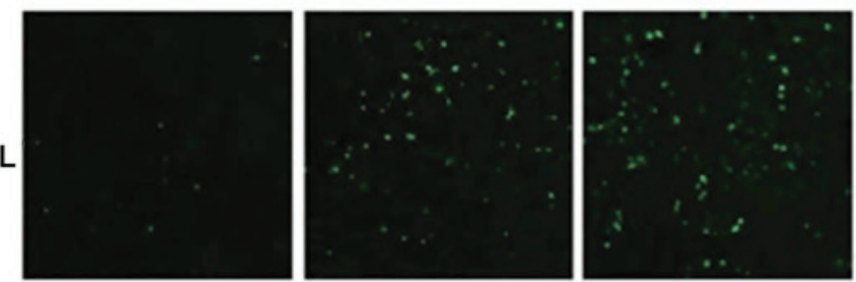

Figure 5. scAAV5-CD40L-M inhibits tumor growth in vivo. Athymic nude mice were xenotransplanted with A549 cells. When $100 \mathrm{~mm}^{3}$-tumors were formed, intratumoral injection with scAAV5 vectors (at $1 \times 10^{11} \mathrm{vgs} /$ mouse) or $0.5 \mathrm{ml}$ phosphate-buffered saline was performed every five days. (A) Tumor growth curves. Treatment of scAAV5-CD40L-M enhanced the inhibition of tumor growth. " $\mathrm{P}<0.05$ vs. scAAV5-GFP. Each point represents the mean \pm standard deviation ( $\mathrm{n}=6$ tumors). (B) Representative image of the various tumors. Mean weights of the tumors were $0.43 \pm 0.03,0.31 \pm 0.02 \mathrm{and} 0.29 \pm 0.03 \mathrm{~g}$ for the scAAV5-GFP, scAAV5-CD40L and scAAV5-CD40L-M groups, respectively. (C) Representative images of tumor sections examined by immunohistochemical staining for CD40L protein expression (magnification, x100). (D) Representative images of tumor sections examined by TUNEL assay. TUNEL-positive cell nuclei (green) were observed under a fluorescence microscope (magnification, x100). scAAV5, self-complementary adeno-associated virus 5; GFP, green fluorescent protein; TUNEL, terminal deoxynucleotidyl transferase-mediated dUTP-fluorescein isothiocyanate nick end-labeling; CD40L-M, CD40 ligand mutant.

potent inducer of apoptosis than the wild-type CD40L, which was in agreement with previous studies by Elmetwali et al (12) and Vardouli et al (17). It is possible that this augmented antitumor activity was a result of 'suicide' and 'fratricide' through constitutive stimulation of CD40 signals by CD40L expression. This antitumor activity was further confirmed in vivo by treatment of pre-existing A549 xenografts without detectable side effects.

In conclusion, the results of the present study demonstrated the direct antitumor effects of scAAV5 delivery of membrane-stable mutant CD40L on human CD40-positive lung carcinomas in vitro and in vivo with few side effects. The balance between the direct cancer growth inhibition of
scAAV-CD40L-M with its immune-activating properties will be examined in forthcoming studies, using a murine, immunocompetent syngeneic cancer model.

\section{Acknowledgements}

The present study was funded by the Priority Academic Program Development of Jiangsu Higher Education Institutions (Jiangsu, China) and supported by grants from the International Science \& Technology Cooperation Program of China (no. 2014DFA31940), the National Natural Science Foundation of China (Beijing, China; nos. 30971320 , 81272602 and 81302014) and the Universities Natural Science 
Research Project of Jiangsu Province (Nanjing, China; no. 12KJB320004).

\section{References}

1. Siegel R, Ma J, Zou Z and Jemal A: Cancer statistics, 2014. CA Cancer J Clin 64: 9-29, 2014.

2. Thomas A and Hassan R: Immunotherapies for non-small-cell lung cancer and mesothelioma. Lancet Oncol 13: e301-e310, 2012.

3. Reck M: What future opportunities may immuno-oncology provide for improving the treatment of patients with lung cancer? Ann Oncol 23 (Suppl 8): viii28-viii34, 2012.

4. Loskog AS and Eliopoulos AG: The Janus faces of CD40 in cancer. Semin Immunol 21: 301-307, 2009.

5. Khong A, Nelson DJ, Nowak AK, et al: The use of agonistic anti-CD40 therapy in treatments for cancer. Int Rev Immunol 31: 246-266, 2012

6. Ullenhag G and Loskog AS: AdCD40L - crossing the valley of death? Int Rev Immunol 31: 289-298, 2012.

7. Vonderheide RH and Glennie MJ: Agonistic CD40 antibodies and cancer therapy. Clin Cancer Res 19: 1035-1043, 2013.

8. Pietravalle F, Lecoanet-Henchoz S, Blasey H, et al: Human native soluble $\mathrm{CD} 40 \mathrm{~L}$ is a biologically active trimer, processed inside microsomes. J Biol Chem 271: 5965-5967, 1996.

9. Georgopoulos NT, Steele LP, Thomson MJ, et al: A novel mechanism of CD40-induced apoptosis of carcinoma cells involving TRAF3 and JNK/AP-1 activation. Cell Death Differ 13: 1789-1801, 2006.

10. Goules A, Tzioufas AG, Manousakis MN, et al: Elevated levels of soluble CD40 ligand (sCD40L) in serum of patients with systemic autoimmune diseases. J Autoimmun 26: 165-171, 2006.

11. Ferroni P, Santilli F, Guadagni F, et al: Contribution of platelet-derived CD40 ligand to inflammation, thrombosis and neoangiogenesis. Curr Med Chem 14: 2170-2180, 2007.

12. Elmetwali T, Young LS and Palmer DH: CD40 ligand-induced carcinoma cell death: a balance between activation of TNFR-associated factor (TRAF) 3-dependent death signals and suppression of TRAF6-dependent survival signals. J Immunol 184: 1111-1120, 2010.

13. Wu JQ, Zhao WH, Li Y, et al: Adeno-associated virus mediated gene transfer into lung cancer cells promoting CD40 ligand-based immunotherapy. Virology 368: 309-316, 2007.
14. Masuta Y, Kato K, Tomihara K, et al: Gene transfer of noncleavable cell surface mutants of human CD154 induces the immune response and diminishes systemic inflammatory reactions. J Immunother 30: 694-704, 2007.

15. Wu J, Zhao W, Zhong L, et al: Self-complementary recombinant adeno-associated viral vectors: packaging capacity and the role of rep proteins in vector purity. Hum Gene Ther 18: 171-182, 2007.

16. Gomes EM, Rodrigues MS, Phadke AP, et al: Antitumor activity of an oncolytic adenoviral-CD40 ligand (CD154) transgene construct in human breast cancer cells. Clin Cancer Res 15: 1317-1325, 2009.

17. Vardouli L, Lindqvist C, Vlahou K, et al: Adenovirus delivery of human CD40 ligand gene confers direct therapeutic effects on carcinomas. Cancer Gene Ther 16: 848-860, 2009.

18. Dzojic H, Loskog A, Tötterman TH and Essand M Adenovirus-mediated CD40 ligand therapy induces tumor cell apoptosis and systemic immunity in the TRAMP-C2 mouse prostate cancer model. Prostate 66: 831-838, 2006.

19. Iida T, Shiba H, Misawa T, et al: Adenovirus-mediated CD40L gene therapy induced both humoral and cellular immunity against rat model of hepatocellular carcinoma. Cancer Sci 99: 2097-2103, 2008.

20. Serba S, Schmidt J, Wentzensen N, et al: Transfection with CD40L induces tumour suppression by dendritic cell activation in an orthotopic mouse model of pancreatic adenocarcinoma. Gut 57: 344-351, 2008.

21. Lindqvist C, Sandin LC, Fransson M and Loskog A: Local AdCD40L gene therapy is effective for disseminated murine experimental cancer by breaking T-cell tolerance and inducing tumor cell growth inhibition. J Immunother 32: 785-792, 2009.

22. von Euler H, Sadeghi A, Carlsson B, et al: Efficient adenovector CD40 ligand immunotherapy of canine malignant melanoma. J Immunother 31: 377-384, 2008.

23. Matthies KM, Newman JL, Hodzic A and Wingett DG: Differential regulation of soluble and membrane CD40L proteins in T cells. Cell Immunol 241: 47-58, 2006.

24. Davies CC, Mason J, Wakelam MJ, et al: Inhibition of phosphatidylinositol 3-kinase- and ERK MAPK-regulated protein synthesis reveals the pro-apoptotic properties of CD40 ligation in carcinoma cells. J Biol Chem 279: 1010-1019, 2004.

25. Dickey DD, Excoffon KJ, Koerber JT, et al: Enhanced sialic acid-dependent endocytosis explains the increased efficiency of infection of airway epithelia by a novel adeno-associated virus. J Virol 85: 9023-9030, 2011.

26. Nonnenmacher M and Weber T: Intracellular transport of recombinant adeno-associated virus vectors. Gene Ther 19: 649-658, 2012. 